\title{
Diferencias de género en la aproximación al cambio de dirección en tenistas profesionales \\ Gender differences in the approach to change of direction in professional tennis players
}

\author{
*Álvaro Durán, **Rafael Martínez-Gallego, *Marc Gimeno
}

*Ypsilon Sport Clinic (España), **Universidad de Valencia (España)

\begin{abstract}
Resumen. El objetivo de este estudio fue medir las velocidades de aproximación a los cambios de dirección (CDD), tras desplazamientos de más de 4 metros, entre hombres y mujeres en el fondo de la pista durante partidos de tenis de élite. Se seleccionó un partido de cada uno de los 5 mejores tenistas masculinos y femeninos del ranking ATP yWTA.Y, de cada partido, 15 CDD. Sobre cada CDD, se aplicó un sistema de seguimiento automático mediante videoanálisis basado en tres puntos corporales (cabeza, tronco y cadera). Se encontraron mayores velocidades en hombres en todos los momentos analizados a partir del penúltimo apoyo (PEN) antes del CDD. También se encontraron diferencias entre ambos sexos en las velocidades parciales durante los pasos previos al CDD. Durante aproximación al CDD, la velocidad de los hombres a partir del antepenúltimo apoyo (ANT) antes del CDD fue aumentada en el PEN y disminuyó en el último apoyo (ULT) antes del CDD, mientras que las mujeres, disminuyeron progresivamente su velocidad a partir del ANT. En conclusión, existen diferencias entre hombres y mujeres en la aproximación a un cambio de dirección, y por tanto en la preparación de golpeos. Por lo tanto, la información proporcionada en este estudio es de vital importancia para el planteamiento de entrenamientos.
\end{abstract}

Palabras clave:Tenis, cambio de dirección, tracking, velocidad

\begin{abstract}
The objective of this study was to measure and compare between genders, speeds of approach to the changes of direction (CDD), after displacements of more than 4 meters in the bottom of the court present in elite tennis. 15 CDDs were selected during a match, from each of the top 5 male and female tennis players in the ATP and WTA rankings. On them, an automatic monitoring system was applied by video analysis based on three body points (head, trunk and hip). Higher velocity values were found for men at all moments analyzed from the penultimate step (PEN) before CDD, as well as substantial differences between both genders in partial velocities during steps prior to CDD. During approach to the CDD, the speed of men from the third to last step before the CDD (ANT) was increased in the PEN and decreased in the last step before the CDD (ULT), while the women progressively decreased their speed from of the ANT. In conclusion, there are many differences between men and women when approaching a change of direction, and therefore in the preparation of blows. Therefore, the information provided in this study is of vital importance for the training approach by coaches and physical trainers.
\end{abstract}

Key words: Tennis, change of direction, tracking, speed.

\section{Introducción}

El tenis es un deporte que goza de una gran popularidad alrededor del mundo, contando con 87 millones practicantes, lo que supone un $1.17 \%$ de la población mundial. De todos estos practicantes, el $47 \%$ son mujeres (International Tennis Federation, 2019). La popularización del tenis promueve una profesionalización diversificada y extensa, dando lugar a un gran número de perfiles técnico-tácticos (Crespo, 2014). De hecho, estudios previos han puesto de manifiesto la importancia de controlar de forma específica las cargas internas,

Fecha recepción: 11-04-21. Fecha de aceptación: 15-09-21

Rafael Martínez-Gallego

Rafael.martinez-gallego@uv.es externas y técnico-tácticas en tenistas de competición (Penalva et al., 2021; Baiget et al., 2019)

Aunque el tenis presenta una gran variabilidad en sus demandas físicas y técnico-tácticas, existen ciertos aspectos que son comunes entre jugadores, entre ellos los cambios de dirección (CDD) realizados durante los puntos. El CDD es un componente del desplazamiento necesario para el desarrollo del juego en el tenis. Durante un partido, los jugadores realizan un promedio de $4 \mathrm{CDD}$ en cada punto, pudiéndose extender esta cifra a más de 15 (Kovacs, 2009). Por tanto, los CDD pueden ser considerados uno de los componentes físicos más importantes para lograr un alto rendimiento en el tenis (Fernández-Fernández et al., 2020). A pesar de esta importancia, la información acerca de los CDD en el tenis es escasa, existiendo muy pocos estudios previos que hayan analizado esta cualidad física (Leone et al., 
2006; Ulbricht et al., 2016), especialmente en jugadores profesionales (Cooke, Quinn, \& Sibte, 2011; Giles, Kovalchik \& Reid, 2020).

El rendimiento en los CDD está influenciado por factores como la técnica, la velocidad de desplazamiento, la capacidad de desaceleración, así como también otras variables relacionadas con la potencia y fuerza musculares (Sheppard \& Young, 2006). Por otra parte, los CDD son ángulo-dependientes (Dos'Santos et al., 2018), de modo que las diferentes situaciones derivadas de la práctica deportiva pueden derivar en diferentes CDD y con ello diferentes requerimientos fisiológicos y mecánicos. De forma específica, en el tenis, algunos estudios han mostrado como las características técnicotácticas y de movimiento se han modificado sustancialmente en los últimos años, poniendo de manifiesto como actualmente los jugadores adoptan estrategias de juego más ofensivas que llevan a una reducción de la duración de los puntos y a grandes velocidades de desplazamiento durante los puntos (Sanz \& Andrade, 2008; Martínez-Gallego et al., 2013; Martínez-Gallego et al., 2017). En el juego de fondo de tenis profesional, más del 70\% de los movimientos se producen de lado a lado de la pista (Wojtys et al., 1996), lo que provoca que los CDD tengan angulaciones cercanas a los $180^{\circ}$. Estos movimientos requieren una frenada sustancial y una serie de pasos previos antes del CDD, con el fin de reducir la velocidad para después acelerar rápidamente (Kovacs, Chandler \& Chandler, 2007). Por tanto, CDD más eficaces y eficientes darán como resultado mejores niveles de equilibrio dinámico, que se traducirán en mejores golpeos y recuperaciones (Kovacs et al., 2008). Por tanto, al ser una cualidad física entrenable, parece lógica la necesidad de incluir los CDD en los entrenamientos, con el objetivo de optimizar los desplazamientos y el control corporal del tenista.

Para que el entrenamiento de los CDD sea específico, es necesario conocer cuales son las características cinemáticas y cinéticas de este tipo de movimientos y, de esa forma, aplicarlos de una forma eficiente en los entrenamientos. Durante la fase de desaceleración previa al $\mathrm{CDD}$, un menor número de pasos requerirá mayores fuerzas de frenada para detener todo el cuerpo, lo que aumentará la eficacia del movimiento y permitirá al jugador o jugadora recorrer una distancia a mayor velocidad. A pesar de que los mecanismos y técnicas asociados a CDD más rápidos estarían en conflicto con mecanismos más seguros (ej., reducción del valgo de rodilla) (Donelon et al., 2020). Con el fin de conocer mejor los mecanismos resultantes de los CDD para ser más eficaces y reducir el riesgo de lesión, han sido estudiados los efectos de niveles altos de fuerza de frenada durante el penúltimo apoyo (PEN) sobre el CDD, destacando su influencia sobre las cargas de la rodilla en el último apoyo y sobre la optimización de fuerzas hacia una nueva dirección (Dos'Santos et al., 2018). Y, observándose diferencias cinéticas y cinemáticas entre hombres y mujeres en el paso previo al CDD (Santos et al., 2020).

A pesar de que la velocidad de aproximación al CDD tiene una gran relación con las fuerzas de frenada y las cargas sobre las articulaciones, ningún estudio previo ha analizado este aspecto en tenistas profesionales. Por tanto, el objetivo de este trabajo es analizar la velocidad de aproximación en los pasos utilizados para decelerar el cuerpo durante un CDD en jugadores y jugadoras profesionales, e identificar las diferencias entre géneros.

\section{Método}

\section{Diseño}

En este estudio se realizó un visionado de partidos de tenis, correspondientes a los cinco mejores jugadores del ranking ATP y las cinco mejores jugadoras del ranking WTA en el momento en que el partido se disputó, en el periodo 2016-2020. Los partidos pertenecían a los campeonatos realizados en Beijing, Montreal, Brisbane, Qatar, Melbourne, Nueva York. Los partidos eran de libre acceso (mediante la plataforma de multimedia «Youtube») y todos se disputaron sobre superficie dura, de acuerdo con la clasificación aportada por la Federación Internacional de Tenis (ITF, 2020). De los jugadores masculinos analizados, tres de los tenistas ejecutaban el revés a dos manos, mientras que en el caso de las jugadoras todas tenían este tipo de revés.

\section{Procedimiento}

Los partidos fueron seleccionados de manera aleatoria, utilizando las grabaciones televisivas. En cada partido, se seleccionaron 15 cambios de dirección que: (A) tuvieran una distancia de más de 4 metros durante el juego de fondo, donde no existe una parada estable, debido a que son potencialmente lesivos (Reid, Quinn, \& Crespo, 2003); y (B) con un ángulo mayor a $60^{\circ}$, que garantizara la necesidad de detenerse por completo antes de avanzar hacia la nueva dirección (Dos'Santos et al., 2018).

El corte de los partidos se utilizó el programa libre de análisis de video LongoMatch (Versión 1.6.0, 
Windows 10 Home 1.9.0.3, Fluendo S.A., Barcelona, España). Para todos los partidos, se estableció el mismo tiempo de inicio $(t=0)$ cuando la pelota entraba en contacto con la raqueta de uno de los dos jugadores durante el primer servicio válido del partido. Estos vídeos fueron exportados con una tasa mínima de 27.5 fotogramas por segundo y una resolución mínima de 720 píxeles, características suficientes para que la observación y posteriores resultados fueran fiables (Barris \& Button, 2008).

El seguimiento de los jugadores se realizó a través del programa libre de análisis de vídeos Kinovea (Versión 0.9.1, Windows 10 Home 1.9.0.3). Este procedimiento fue llevado a cabo por un único observador, el cual fue el único partícipe del proceso de seguimiento que fue realizado utilizando siempre la misma computadora. En primer lugar, se creó una cuadrícula adaptada al plano de la cámara que cubría la superficie de juego delimitada en el primer fotograma del vídeo. A la cuadrícula se le aplicaron las medidas reglamentarias de ancho $(10.97 \mathrm{~m})$ y de largo $(23.77 \mathrm{~m})$, de acuerdo con la normativa de la ITF (2002). Una vez establecida la superficie de juego, se pasó al seguimiento de los jugadores (Figura 1). El seguimiento se realizó mediante tres puntos corporales: cabeza (A), zona media de la espalda o tronco (B) y cadera (C), permitiendo una menor oscilación con respecto a las extremidades (Barris y Button, 2008).

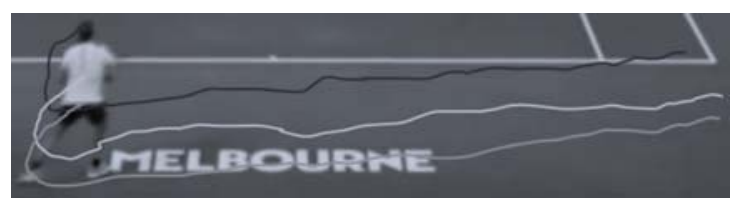

Figura 1. Seguimiento de la trayectoria del jugador durante el juego de fondo.

El seguimiento se inició en el «split step», tras el golpeo y recuperación, y finalizó en el momento en el que uno de los dos pies entraba en contacto con el pavimento tras el CDD (Kovacs, 2014). Durante el seguimiento, los datos aportados por el sistema automático fueron ajustados manualmente en caso de error (Figura 2). A partir de las trayectorias, se extrajeron variables relacionadas con la velocidad, aceleración, posición y distancia total.

Por último, se seleccionaron los fotogramas correspondientes al instante en el que el pie entraba en contacto con el pavimento para los últimos tres apoyos del CDD (Dos'Santos et al., 2017): antepenúltimo apoyo (ANT), penúltimo apoyo (PEN) y último apoyo (ULT). Todos estos datos fueron almacenados de forma manual en una hoja de cálculo Excel (Versión 16.36, MacOS Catalina 10.15.4, Microsoft Corporation, Redmond,

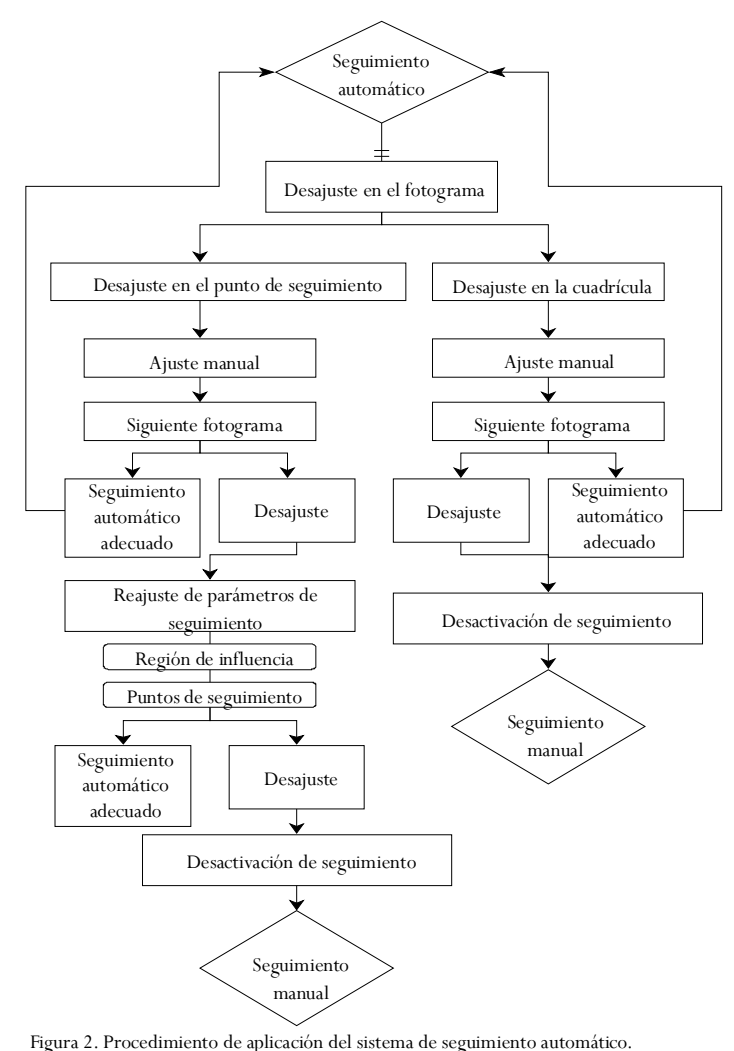

USA).

\section{Análisis estadístico}

Los datos fueron importados en el programa de análisis estadístico Jamovi (Version 1.1.9, MacOS Catalina 10.15.4). Se utilizó la prueba de normalidad Shapiro Wilk para todas las variables y, considerando que los datos relativos a la mayoría de estas no presentaban una distribución normal, se analizó mediante estadística no paramétrica. Por tanto, se utilizó la U de Mann-Whitney y tamaños de efectos ( $\mathrm{d}$ de Cohen) para evaluar las diferencias entre los jugadores masculinos y femeninos. Los tamaños de efecto se interpretaron como triviales $(<0,19)$, pequeños $(0,20-0,59)$, moderados $(0,60-$ $1,19)$, grandes $(1,20-1,99)$ y muy grandes $(2,0-4,0)$ (Hopkins, 2002). El criterio de significación fue establecido en $\mathrm{p} d » 0.05$.

\section{Resultados}

Durante la fase anterior al CDD, como se puede apreciar en la Figura 3 a partir de la observación de la mediana (Mn) y el rango intercualtílico (RI), la velocidad $(\mathrm{m} / \mathrm{s})$ de los hombres a partir del ANT mantuvieron la velocidad en PEN y la disminuyeron en ULT. En cambio, las mujeres disminuyeron la velocidad del ANT al PEN y continuaron reduciéndola en ULT. 


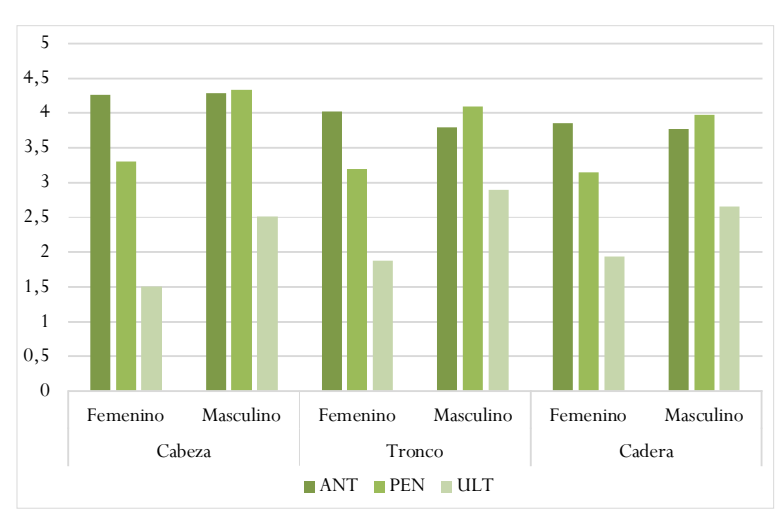

Figura 3. Evolución de la velocidad en hombres y mujeres durante los apoyos previos al cambio de dirección.

La Tabla 3 muestra que, durante el ANT, no existieron diferencias significativas entre hombres y mujeres en las mediciones realizadas en la cabeza $(\mathrm{p}=0,37)$, tronco $(p=0,5)$ y la cadera $(p=0,66)$. Durante el PEN se encontraron menores velocidades en mujeres respecto a los hombres: cabeza $(\mathrm{p}<, 001 ; \eta 2=0,16)$; tronco $(\mathrm{p}<$ $, 001 ; \eta 2=0,19) ; y$ cadera $(p<, 001 ; \eta 2=0,16)$. En el ULT, los hombres obtuvieron mayores velocidades que las mujeres en cabeza $(\mathrm{p}<.001 ; \eta 2=0,15)$, tronco $(\mathrm{p}<$ $.001 ; \eta 2=0,13)$ y cadera $(\mathrm{p}<.001 ; \eta 2=0,097)$. La velocidad de salida de los hombres fue mayor que las mujeres en cabeza $(\mathrm{p}<.001 ; \eta 2=0,17)$, tronco $(\mathrm{p}<, 001$; $\eta 2=0,102)$ y cadera $(\mathrm{p}<, 001 ; \eta 2=0,082)$.

Tabla 1.

Análisis descriptivo y estadístico de velocidades parciales (m/s) durante ANT, PEN, ULT y SAL.

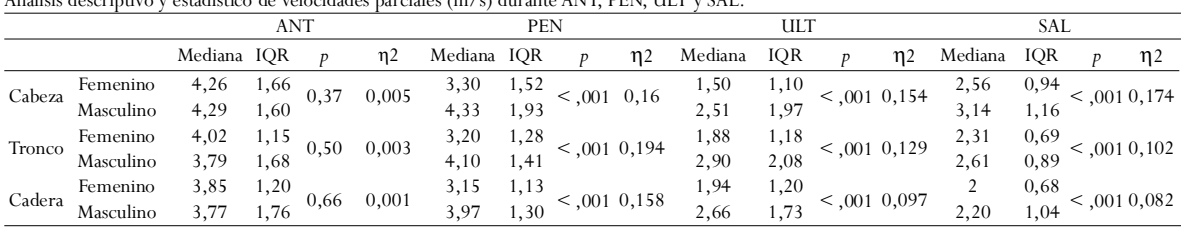

\section{Discusión}

Varios estudios realizados en deportes de raqueta muestran que, en líneas generales, los hombres se mueven a mayor velocidad que las mujeres (Galé-Ansodi, 2014; Rodríguez, 2012). Ellenbecker, Pluim, Vivier y Sniteman (2009), llevaron a cabo una revisión de estudios que muestra que la región que los jugadores de tenis se lesionan con más frecuencia es la de las extremidades inferiores (rango de 39-65\%), seguido de las extremidades superiores (rango de $24-46 \%$ ) y la cabe$\mathrm{za} /$ tronco (rango de 8-22\%). A pesar de ello, las diferencias fisiológicas y físicas existentes entre ambos sexos (París, 2000), pueden dar como resultado diferencias en la fluctuación de la velocidad durante la fase de frenada hacia el CDD. Los resultados de nuestro estudio muestran que las mujeres reducen la velocidad de desplazamiento en PEN y continúan reduciéndola en ULT, por el contrario, los hombres no disminuyen su velocidad con respecto a ANT y la disminuyen del PEN al ULT. Esto nos indica que las mujeres frenan durante los últimos tres apoyos, mientras que los hombres durante los últimos dos, con lo cual, aplican mayores niveles de fuerza que las mujeres. Estos hechos coinciden con los resultados de un estudio realizado por Spiteri et al. (2014), donde se mostró que los hombres producían movimientos con mayor rapidez, derivados de una mayor fuerza, impulso y ángulos de flexión de tronco y rodilla en comparación con las mujeres, aunque estas son variables profundamente dependientes de la experiencia, del ranking, o del estilo de juego (McLean et al., 2004).

Un estudio realizado por Sigward et al. (2015), muestra un patrón similar en los datos de fuerzas reactivas verticales y posteriores contra el pavimento entre sexos en cambios de dirección de entre $45^{\circ}$ y $115^{\circ}$. Durante los CDD más cerrados, las mujeres exhibieron mayores picos de valgo de rodilla y una mayor carga en el plano frontal de la rodilla. Por otro lado, comprobaron que tanto hombres como mujeres producen una cantidad de fuerza vertical por kilogramo similar en los CDD $135^{\circ}$ y $180^{\circ}$ (Schreurs et. al., 2017). Sin embargo, estos mismos autores vieron que el momento de fuerza por kilogramo en la relación varo/valgo fue mayor en mujeres cuanto más agudo era el CDD. Además, observaron mayores momentos de fuerza de flexión del cuádriceps en mujeres que hombres en $135^{\circ}$ pero similares en $180^{\circ}$. En esta misma línea, otros autores comprobaron en mujeres que la fuerza excéntrica se relacionaba con mayores reducciones en la velocidad entre el penúltimo y el último y con una mayor velocidad en la realización en los cambios de dirección (Jones et. al., 2017). Finalmente, Dos'Santos et. al. (2018) vieron que aquellos atletas que realizaban los CDD más rápidos eran los que mayores fuerzas de frenado realizaban en el penúltimo respecto al último paso y menor tiempo de contacto tenían en el último paso.

Por tanto, un aumento en los pasos de frenado podría ser una estrategia para optimizar los cambios de dirección, lo que estaría de acuerdo con la revisión realizada por Dos'Santos et. al. (2019) donde destacan la importancia de la desaceleración en los pasos previos como forma de optimizar el CDD. Esta sería una estra- 
tegia con el fin de adaptar las elevadas fuerzas producidas generada por el desplazamiento a la capacidad del deportista y, por ende, reducir el riesgo de lesión.

\section{Aplicaciones prácticas}

Este es un estudio pionero en la utilización de seguimiento para identificar características de los cambios de dirección en tenistas. A partir de los resultados obtenidos en este estudio, se anima a desarrollar la capacidad de generar fuerzas a altas velocidades en miembros inferiores según las necesidades y estrategias de los deportistas. Debido a la importancia de la velocidad de juego en pavimentos duros, la rápida aplicación de fuerzas de aceleración y frenada en tiempos de contacto puede llegar a ser decisiva durante el juego del tenis. En este sentido, el presente estudio podría extraerse que mediante herramientas de bajo coste es posible inferir déficits de en la producción de fuerza y objetivar estrategias de desaceleración a través del análisis de los CDD. Además, es importante analizar los CDD durante los partidos y fuera de ellos con el fin de prescribir un ejercicio adecuado para mejorar en caso de deficiencia.

Centrándonos en los resultados del estudio, los hombres deberían enfatizar su trabajo en reducir el impacto sobre el último apoyo procurando frenar con antelación, mientras que las mujeres se deberían centrar el trabajo de fuerza para desplazar la frenada al penúltimo paso, lo que resultaría en unos mayores valores de velocidad durante los desplazamientos. Con estos objetivos de entrenamiento, derivados de los resultados de el presente estudio, se lograría optimizar la frenada y reducir el riesgo de lesiones durante los CDD.

\section{Conclusión}

Los resultados obtenidos a partir de este estudio muestran que, para cambios de dirección de más de 4 metros, donde no existe una parada estable, las mujeres comienzan a frenar antes de un CDD un paso antes que los hombres, obteniendo valores de velocidad en el último apoyo significativamente más bajos que estos últimos. Este hecho permite que la jugadora disponga de mayor tiempo para colocarse y que se reduzca significativamente el impacto de la frenada en miembros inferiores.

\section{Limitaciones}

La principal limitación de este estudio la encontra- mos en los videos, ya que los planos tenían movimiento y las referencias debían ser ajustadas constantemente.Y, por otro lado, la velocidad de grabación.

\section{Referencias}

Baiget, E., Iglesias, X., Fuentes, J. P., \& Rodriguez, F. A. (2019). New Approaches for On-court Endurance Testing and Conditioning in Competitive Tennis Players. Strength and Conditioning Journal, 41(5), 916.

Barris, S., \& Button, C. (2008). A review of visionbased motion analysis in sport. Sports Medicine, 38(12), 1025-1043.

Cooke, K., Quinn, A., \& Sibte, N. (2011). Testing speed and agility in elite tennis players. Strength \& Conditioning Journal, 33(4), 69-72.

Crespo, M. (2014). Las profesiones del mundo del tenis. E-coach. Revista Electrónica del Técnico de Tenis, 20, 6-14.

Cross, R. (2014). El tenis masculino vs. el tenis femenino. ITF Coaching and Sport Science Review, 22(62), 3-5.

Dos'Santos, T., Thomas, C., Jones, P. A., \& Comfort, P. (2017). Mechanical Determinants of Faster Change of Direction Speed Performance in Male Athletes. Journal of Strength and Conditioning Research, 31(3), 696-705.

Dos'Santos, T., Thomas, C., Comfort, P., \& Jones, P. A. (2018). The effect of angle and velocity on change of direction biomechanics: An angle-velocity trade-off. Sports medicine, 48(10), 2235-2253.

Ellenbecker, T.S., Pluim, B., Vivier, S. \& Sniteman, C. (2009). Common injuries in tennis players: exercises to address muscular imbalances and reduce injury risk. Strength \& Conditioning Journal, 31, 50-58.

Fernandez-Fernandez, J., Loturco, I., Pereira, L. A., Del Coso, J., Areces, F., Gallo-Salazar, C., \& Sanz-Rivas, D. (2020). Change of Direction Performance in Young Tennis Players: A Comparative Study Between Sexes and Age Categories. Journal of strength and conditioning research. Ahead of print.

Galé-Ansodi, C. (2014). Youths tennis players ' velocity and acceleration in match play. Revista Internacional de Los Deportes Colectivos, 18, 50-65.

Giles, B., Kovalchik, S., \& Reid, M. (2020). A machine learning approach for automatic detection and classification of changes of direction from player tracking data in professional tennis. Journal of sports sciences, 38(1), 106-113.

International Tennis Federation. (2019). Global tennis 
report. Recuperado de: www.itftennis.com.

Kovacs, M., Chandler, W. B., \& Chandler, T. J. (2007). Tennis training enhancing on-court performance. Racquet Tech Publishing. Vista, CA.

Kovacs, M. S., Roetert, E. P., \& Ellenbecker,T. S. (2008). Efficient deceleration:The forgotten factor in tennisspecific training. Strength and Conditioning Journal, 30(6), 58-69.

Kovacs, M. S. (2009). Movement for tennis: The importance of lateral training. Strength and Conditioning Journal, 31(4), 77-85.

Leone, M., Comtois, A. S., Tremblay, F., \& Léger, L. (2006). Specificity of running speed and agility in competitive junior tennis players. Med Sci Tennis, 11, 10-11.

Martínez-Gallego, R., Guzmán, J. F., Crespo, M., Ramón-Llin, J., \& Vuèkoviæ, G. (2017). Technical, tactical and movement analysis of men's professional tennis on hard courts. The Journal of sports medicine and physical fitness, 59(1), 50-56.

Martínez-Gallego, R., Guzmán, J. F., James, N., Pers, J., Ramón-Llin, J., \& Vuckovic, G. (2013). Movement characteristics of elite tennis players on hard courts with respect to the direction of ground strokes. Journal of sports science \& medicine, 12(2), 275.

McLean, S. G., Lipfert, S. W., \& Van Den Bogert, A. J. (2004). Effect of gender and defensive opponent on the biomechanics of sidestep cutting. Medicine and Science in Sports and Exercise, 36(6), 1008-1016.

París, C. L. (2000). Influencia del sexo en la práctica deportiva. Biología de la mujer

deportista. Arbor, 165(650), 249-263.

Penalva, F., Guzmán, J. F., Martínez-Gallego, R., \& Crespo, M. (2021). Design and validation of a tennis tool to control on-court technical and tactical training content. International Journal of Sports Science \& Coaching, 17479541211027428.

Reid, M., Quinn, A., \& Crespo, M. (2003). Fuerza y condición física para el tenis. ITF Ltd. Londres.

Rodríguez, A. C. (2012). El pádel de competición. Análisis de las respuestas fisiológicas, físicas y psicológicas (Doctoral dissertation, Universidad de Málaga).

Sanz, D. y Andrade, J. C. (2008). Entrenamiento del juego ofensivo para jugadores de competición. ECoach, 2, 1-4.

Sheppard, J. M., \& Young,W. B. (2006). Agility literature review: Classifications, training and testing. Journal of sports sciences, 24(9), 919-932.

Sigward, S. M., Cesar, G. M., \& Havens, K. L. (2015). Predictors of frontal plane knee moments during side-step cutting to 45 and 110 degrees in men and women: Implications for anterior cruciate ligament injury. Clinical Journal of Sport Medicine, 25(6), 529534.

Spiteri, T., Hart, N. H., \& Nimphius, S. (2014). Offensive and defensive agility: A sex comparison of lower body kinematics and ground reaction forces. Journal of Applied Biomechanics, 30(4), 514-520.

Ulbricht, A., Fernandez-Fernandez, J., MendezVillanueva, A., \& Ferrauti, A. (2016). Impact of fitness characteristics on tennis performance in elite junior tennis players. The journal of strength \& conditioning research, 30(4), 989-998.

Wojtys, E. M., Huston, L. J., Taylor, P. D., \& Bastian, S. D. (1996). Neuromuscular adaptations in isokinetic, isotonic, and agility training programs. American Journal of Sports Medicine, 24(2), 187-192.

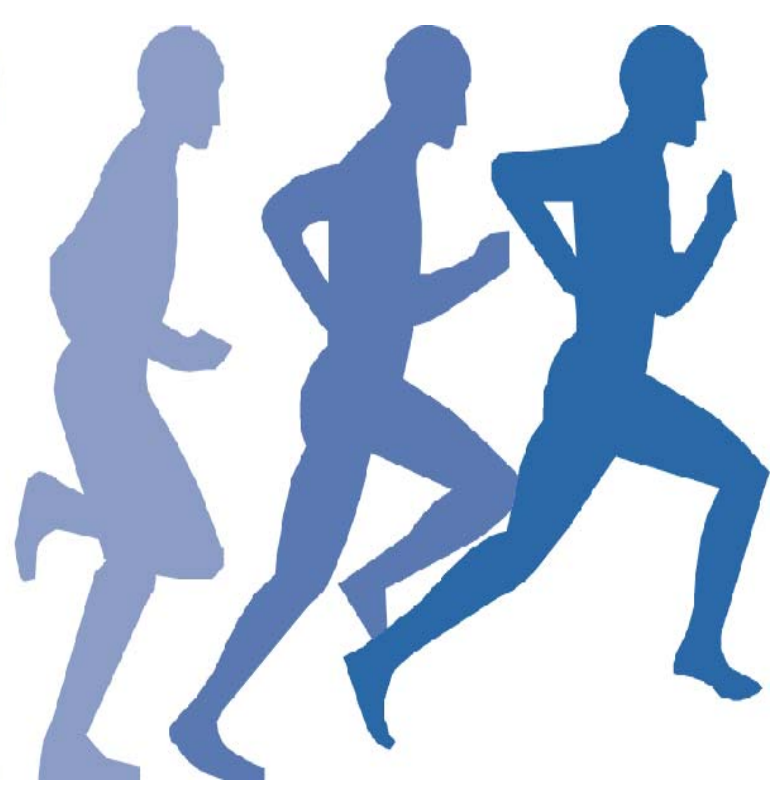

